\title{
FINITE-DIFFERENCE IMPLEMENTATION OF CONSERVED PROPERTIES OF VECTOR NLSE
}

\author{
MICHAIL TODOROV
}

Communicated by Jean-Francois Ganghoffer

\begin{abstract}
In this work we consider conserved properties of the vector Nonlinear Schrödinger Equations for linearly polarized solitons in the initial configuration. We derive analytic formulae for the mass, pseudomomentum and energy and compare results with the discrete formulae based on a conservative fully implicit finite-difference scheme in complex arithmetic.
\end{abstract}

\section{Introduction}

The investigation of soliton supporting systems is of great importance both in view of applications and for the fundamental understanding of the phenomena associated with propagation of solitons. Recently, elaborate models such as vector Nonlinear Schrödinger Equation (NLSE) appeared in the literature (see, for example $[4,6]$ ). They involve more parameters and have richer phenomenology but, as a rule, they are not fully integrable and require numerical approaches. The nonfully-integrable models possess as a rule three conservation laws: (wave) "mass", (wave) momentum, and energy and these have to be faithfully represented by the numerical schemes.

An implicit scheme of Crank-Nicolson type was first proposed for the single NLS in the extensive numerical treatise [9]. The concept of the internal iterations was first applied to vector NLSE in [3] and extended in [7] in order to ensure the implementation of the conservation laws on difference level within the round-off error of the calculations. Here, we follow generally the works [3,7] but focus on the conservative properties of the conservative scheme.

The comparison of both analytic and numerical calculations shows a significant advantage in the efficiency of the finite difference scheme and algorithm. 


\section{Vector Nonlinear Schrödinger Equation}

In optics, the most popular model is the cubic Schrödinger equation which describes the single-mode wave propagation in a fiber $[1,2]$. It has the form

$$
\mathrm{i} \psi_{t}+\beta \psi_{x x}+\alpha|\psi|^{2} \psi=0
$$

where $\mathrm{i}=\sqrt{-1}$ and $\psi(x, t)$ is a complex-valued wave function. Depending on the sign of coefficient $\alpha$, the localized solutions of equation (1) are either the hyperbolic secants ("bright solitons") or hyperbolic tangents ("dark solitons"). Since the fibers also allow propagation of multiple "orthogonal" modes, a multi-component version of equation (1) has been actively investigated during the last decade.

A general form of the vector Nonlinear Schrodinger Equation (NLSE) reads

$$
\begin{aligned}
& \mathrm{i} \psi_{t}=\beta \psi_{x x}+\left(\alpha_{1}|\psi|^{2}+\left(\alpha_{1}+2 \alpha_{2}\right)|\phi|^{2}\right) \psi+\gamma \psi+\Gamma \phi=0 \\
& \mathrm{i} \phi_{t}=\beta \phi_{x x}+\left(\alpha_{1}|\phi|^{2}+\left(\alpha_{1}+2 \alpha_{2}\right)|\psi|^{2}\right) \phi+\gamma \phi+\Gamma \psi=0
\end{aligned}
$$

where $\beta$ is the dispersion coefficient and $\alpha_{1}$ describes the self-focusing of a signal for pulses in birefringent media. Complex-valued coefficients $\gamma$ and $\Gamma$ are responsible for the linear coupling between the two equations. Coefficient $\alpha_{2}$ governs the nonlinear coupling between the equations. It is interesting to note that when $\alpha_{2}=0$, the nonlinear coupling is not present despite the fact that "cross-terms" proportional to $\alpha_{1}$ appear in the equations. In fact, when $\gamma=\Gamma=\alpha_{2}=0$, the solution of the two equations are identical, $\psi \equiv \phi$, and equal to the solution of single NLSE, equation (1) with nonlinearity coefficient $\alpha=2 \alpha_{1}$. The coefficient $\alpha_{2}$ is called sometimes "cross-phase modulation" and its value when $\alpha_{2} \neq 0$ plays the role of defining the elliptic, circular and linear polarizations. In this case, integrability is lost, and numerical methods are to be used to study the evolution of the system.

Here is to be mentioned that two main versions of equation (1) appear in the literature. In the first one, the sign of the time derivative is positive (as in equation (1), which is the most popular version in nonlinear optics), and in the other one the sign is negative. Unlike the parabolic equations, changing the sign does not make the equation incorrect in the sense of Hadamard. Hence, it does not really make difference which version will be used.

Functions $\psi$ and $\phi$ have various interpretations in the context of optic pulses, including the amplitudes of $x$ and $y$ polarizations in a birefringent nonlinear planar waveguide, pulsed wave amplitudes of left and right circular polarizations, etc. The quantity $\gamma$ is called normalized birefringence, and $\Gamma$ is the relative propagation constant. The presence of the two new parameters, $\gamma$ and $\Gamma$, in equations (2) makes the 
phenomenology of the system (2) much richer. In particular, they allow to study the phenomena such as "self-dispersion", "cross-dispersion", and dissipation, etc. (see [7] and the literature cited therein).

For $\Gamma=\gamma=0$, equation (2) is alternatively called the Gross-Pitaevskii equation or an equation of Manakov-Type. It was solved analytically for the case $\alpha_{2}=0, \beta=$ $\frac{1}{2}$ by Manakov [5] via inverse scattering transform, who generalized an earlier result by Zakharov and Shabat $[12,13]$ for the scalar cubic NLSE (i.e., equation $(2)_{\psi}$ with $\left.\phi(x, t)=0\right)$.

Let us define the mass $M$, the (pseudo)momentum $P$, and the energy $E$, of the wave system as follows

$$
\begin{aligned}
& M \stackrel{\text { def }}{=} \frac{1}{2 \beta} \int_{-\infty}^{\infty}\left(|\psi|^{2}+|\phi|^{2}\right) \mathrm{d} x, P \stackrel{\text { def }}{=}-\int_{-\infty}^{\infty} \Im\left(\psi \bar{\psi}_{x}+\phi \bar{\phi}_{x}\right) \mathrm{d} x, E \stackrel{\text { def }}{=} \int_{-\infty}^{\infty} \mathcal{H} \mathrm{d} x \\
& \mathcal{H} \stackrel{\text { def }}{=} \beta\left(\left|\psi_{x}\right|^{2}+\left|\phi_{x}\right|^{2}\right)-\frac{\alpha_{1}}{2}\left(|\psi|^{4}+|\phi|^{4}\right) \\
& \quad-\left(\alpha_{1}+2 \alpha_{2}\right)\left(|\phi|^{2}|\psi|^{2}\right)-\gamma\left(|\psi|^{2}+|\phi|^{2}\right)-2 \Gamma \Re(\bar{\phi} \psi)
\end{aligned}
$$

where $\mathcal{H}$ is the Hamiltonian density of the system. Note that the factor $\frac{1}{2 \beta}$ is a matter of definition and is added for the sake of further convenience. In the same vein, the signs in the expression of the energy are up to a definition and the choice in the present paper is based on considerations of further convenience when the quasi-particles are considered. It is readily proved that these quantities are either conserved on the solutions of equation (2), or a balance law holds, namely

$$
\frac{\mathrm{d} M}{\mathrm{~d} t}=0, \quad \frac{\mathrm{d} P}{\mathrm{~d} t}=\left.\mathcal{H}\right|_{x=L_{2}}-\left.\mathcal{H}\right|_{x=-L_{1}}, \quad \frac{\mathrm{d} E}{\mathrm{~d} t}=0
$$

where $-L_{1}$ and $L_{2}$ are the left end and the right end of the interval under consideration. For asymptotic boundary conditions, the requirement $\psi, \phi=0$ at infinity entails the requirement that the spatial derivatives also vanish. As a result, the Hamiltonian density vanishes at infinity and the balance law for the pseudomomentum becomes a conservation law.

We assume that for each of the functions $\phi, \psi$ the initial condition is of the form of a single propagating soliton, namely

$$
\begin{aligned}
\psi(x, t), \phi(x, t) & =A \operatorname{sech}[b(x-X-c t)] \exp \left[\mathrm{i}\left(\frac{c}{2 \beta}(x-X)-n t\right)\right] \\
b^{2} & =\frac{1}{\beta}\left(n+\gamma+\frac{c^{2}}{4 \beta}\right), \quad A=b \sqrt{\frac{2 \beta}{\alpha_{1}}}, \quad u_{c}=\frac{2 n \beta}{c}
\end{aligned}
$$


where $X$ is the spatial position (center of soliton) where the modulus soliton has a maximum, $c$ is the phase speed, and $n$ is the carrier frequency. Respectively $b^{-1}$ is a measure of the support of the localized wave.

In this paper we investigate the evolution of systems of waves which at initial of time are superpositions of solitons of type of equation (5) and which evolve according to system of equations (2).

To solve this problem numerically, we use the conservative scheme in complex arithmetic described in [10]. If one is to construct a numerical algorithm, the above conservation laws have to be embodied in the scheme in order to faithfully represent the physics of the problem. We use different number of points in spatial direction, typically of order between 8000-20000 points.

The parametric space of the problem is multidimensional, and it is impossible to exhaust the different ranges in a single paper. We focus our attention here on the effect of the nonlinear coupling and set $\Gamma=\gamma=0$. We also fix $\beta=1$, because, in fact, the independent variable $x$ can be scaled by $\beta$ and the latter is not an independent parameter. For the predominant set of numerical experiments, we choose initial solitons which are moving envelopes over standing wave, i.e., $n=0$. Similarly to the dispersion parameter $\beta$, the nonlinearity parameter $\alpha$ can be absorbed in the amplitude of the solitons and can be held fixed. Thus, the parameter to be varied is $\alpha_{2}$, and more specifically, its ratio to $\alpha_{1}$. For definiteness, we fix $\alpha_{1}=1$.

The aim of our work is to better understand the particle-like behavior and properties of the localized waves. We call a localized wave a quasi-particle (QP) if it survives the collision with other QPs (or some other kind of interactions) without losing its identity.

\section{Analytic Expressions for Conserved Properties}

Let us assume here that the two components of the vector soliton are moving together without changing their relative position. Then for both $\psi$ and $\phi$ components we have the same phase speed $c$, but the amplitudes $A$, the size of support $b$, and the frequencies $n$ can be different. A propagating soliton of this type is described by the following formulas

$$
\begin{aligned}
& \psi=A_{\psi} \operatorname{sech}\left[b_{\psi}(x-c t)\right] \exp \left[\mathrm{i}\left(\frac{c}{2 \beta} x-n_{\psi} t\right)\right] \\
& \phi=A_{\phi} \operatorname{sech}\left[b_{\phi}(x-c t)\right] \exp \left[\mathrm{i}\left(\frac{c}{2 \beta} x-n_{\phi} t\right)\right] .
\end{aligned}
$$


When the compound solution, the vector $(\psi, \phi)$, propagates steadily, the above parameters are related as in equation (5).

For a solution of type equation (6) the mass is given by the following formula

$$
\begin{aligned}
M= & \frac{1}{2 \beta} \int_{-\infty}^{\infty}\left(|\psi|^{2}+|\phi|^{2}\right) \mathrm{d} x=\frac{1}{2 \beta} A_{\psi}^{2} \int_{-\infty}^{\infty} \operatorname{sech}^{2}\left[b_{\psi}(x-c t)\right] \mathrm{d} x \\
& +\frac{1}{2 \beta} A_{\phi}^{2} \int_{-\infty}^{\infty} \operatorname{sech}^{2}\left[b_{\phi}(x-c t)\right] \mathrm{d} x=\left.\frac{1}{2 \beta} \frac{A_{\psi}^{2}}{b_{\psi}} \tanh \left[b_{\psi}(x-c t)\right]\right|_{-\infty} ^{\infty} \\
& +\left.\frac{1}{2 \beta} \frac{A_{\phi}^{2}}{b_{\phi}} \tanh \left[b_{\phi}(x-c t)\right]\right|_{-\infty} ^{\infty}=\frac{1}{\beta}\left(\frac{A_{\psi}^{2}}{b_{\psi}}+\frac{A_{\phi}^{2}}{b_{\phi}}\right) .
\end{aligned}
$$

For the wave momentum (pseudomomentum), we get in a similar fashion

$$
\begin{aligned}
P & =\int_{-\infty}^{\infty} \Im\left[\psi \bar{\psi}_{x}+\phi \bar{\phi}_{x}\right] \mathrm{d} x \\
& =\int_{-\infty}^{\infty}\left(\frac{A_{\psi}^{2} c}{2 \beta} \operatorname{sech}^{2}\left[b_{\psi}(x-c t)\right]+\frac{A_{\phi}^{2} c}{2 \beta} \operatorname{sech}^{2}\left[b_{\phi}(x-c t)\right]\right) \mathrm{d} x \\
& =\frac{1}{\beta}\left(\frac{A_{\psi}^{2}}{b_{\psi}}+\frac{A_{\phi}^{2}}{b_{\phi}}\right) c \equiv M c .
\end{aligned}
$$

The momentum of the quasi-particle is exactly the product of the mass and the phase speed.

Now, for the energy we get

$$
\begin{aligned}
E= & \int_{-\infty}^{\infty}\left[\beta\left(\left|\psi_{x}\right|^{2}+\left|\phi_{x}\right|^{2}\right)-\frac{1}{2} \alpha_{1}\left(|\psi|^{4}+|\phi|^{4}\right)\right. \\
& \left.-\left(\alpha_{1}+2 \alpha_{2}\right)\left(|\phi|^{2}|\psi|^{2}\right)-\gamma\left(|\psi|^{2}+|\phi|^{2}\right)-2 \Gamma \mathcal{R}(\bar{\psi} \psi)\right] \mathrm{d} x
\end{aligned}
$$




$$
\begin{aligned}
= & \frac{2}{3} \beta\left(A_{\psi}^{2} b_{\psi}+A_{\phi}^{2} b_{\phi}\right)+\frac{c^{2}}{2 \beta}\left(\frac{A_{\psi}^{2}}{b_{\psi}}+\frac{A_{\phi}^{2}}{b_{\phi}}\right)-\frac{2}{3} \alpha_{1}\left(\frac{A_{\psi}^{4}}{b_{\psi}}+\frac{A_{\phi}^{4}}{b_{\phi}}\right) \\
& -\left(\alpha_{1}+2 \alpha_{2}\right) A_{\psi}^{2} A_{\phi}^{2} \int_{-\infty}^{\infty} \operatorname{sech}^{2}\left[b_{\psi}(x-c t)\right] \operatorname{sech}^{2}\left[b_{\phi}(x-c t)\right] \mathrm{d} x \\
& -2\left[\gamma\left(\frac{A_{\psi}^{2}}{b_{\psi}}+\frac{A_{\phi}^{2}}{b_{\phi}}\right)+2 \Gamma \frac{A_{\psi}^{2}}{b_{\psi}}\right] .
\end{aligned}
$$

In the above formula one of the integrals cannot be found analytically directly unless $b_{\psi}=b_{\phi}$. Yet, we can have a reasonable approximation after noting that

$$
\begin{aligned}
\operatorname{sech}^{2}\left[b_{\psi}(x-c t)\right] \operatorname{sech}^{2}\left[b_{\phi}(x-c t)\right] & 4 \\
& =\frac{4}{\left\{\cosh \left[\left(b_{\psi}+b_{\phi}\right)(x-c t)\right]+\cosh \left[\left(b_{\psi}-b_{\phi}\right)(x-c t)\right]\right\}^{2}}
\end{aligned}
$$

and additionally for the cases treated in this work, $b_{\psi} \approx b_{\phi}$, i.e., $\left|b_{\phi}-b_{\psi}\right| \ll$ $\left|b_{\phi}+b_{\psi}\right|$. In such a case, in the region where $\cosh \left[\left(b_{\psi}-b_{\phi}\right)(x-c t)\right]$ changes rapidly, one can assume that $\cosh \left[\left(b_{\psi}-b_{\phi}\right)(x-c t)\right] \sim 1$. Then

$$
\begin{aligned}
\int_{-\infty}^{\infty} & \frac{4 \mathrm{~d} x}{\left\{\cosh \left[\left(b_{\psi}+b_{\phi}\right)(x-c t)\right]+\cosh \left[\left(b_{\psi}-b_{\phi}\right)(x-c t)\right]\right\}^{2}} \\
& \approx \int_{-\infty}^{\infty} \frac{4 \mathrm{~d} x}{\left[1+\cosh \left(b_{\psi}+b_{\phi}\right)(x-c t)\right]^{2}} \\
& =\int_{-\infty}^{\infty} \frac{\mathrm{d} x}{\cosh ^{4}\left[\frac{1}{2}\left(b_{\psi}+b_{\phi}\right)(x-c t)\right]}=\frac{8}{3} \frac{1}{b_{\psi}+b_{\phi}} .
\end{aligned}
$$

The accuracy of this formula can be easily verified for a couple of specific values of $b_{\psi}$ and $b_{\phi}$ for which the original integral can also be found analytically. For specific ratios $b_{\phi} / b_{\psi}$, both the approximate and exact integrals can be represented as $\kappa / b_{\psi}$, where $\kappa$ is a different coefficient for the different cases. The results for several different ratios $b_{\phi} / b_{\psi}$ show that up to $b_{\phi}=2 b_{\psi}$ the approximation is very reasonable - it does not exceed $3.5 \%$, and hence can be used to get an approximate analytical expression for the integral and compare the energy to the numerically obtained value.

Finally, under the above assumption that the scales of the supports for the two components are not very different, we have the following analytical approximation 
of the energy

$$
\begin{aligned}
E \approx & \frac{c^{2}}{2}\left[\frac{1}{\beta}\left(\frac{A_{\psi}^{2}}{b_{\psi}}+\frac{A_{\phi}^{2}}{b_{\phi}}\right)\right]+\frac{2}{3} \beta\left(A_{\psi}^{2} b_{\psi}+A_{\phi}^{2} b_{\phi}\right)-\frac{2}{3} \alpha_{1}\left(\frac{A_{\psi}^{4}}{b_{\psi}}+\frac{A_{\phi}^{4}}{b_{\phi}}\right) \\
& -\frac{8}{3}\left(\alpha_{1}+2 \alpha_{2}\right) \frac{A_{\psi}^{2} A_{\phi}^{2}}{b_{\psi}+b_{\phi}}-2\left[\gamma\left(\frac{A_{\psi}^{2}}{b_{\psi}}+\frac{A_{\phi}^{2}}{b_{\phi}}\right)+2 \Gamma \frac{A_{\psi}^{2}}{b_{\psi}}\right] .
\end{aligned}
$$

The term $\frac{c^{2}}{2}\left[\frac{1}{\beta}\left(\frac{A_{\psi}^{2}}{b_{\psi}}+\frac{A_{\phi}^{2}}{b_{\phi}}\right)\right] \equiv \frac{M c^{2}}{2}$ can be called the "kinetic energy" of the quasi-particle, while the rest of the terms can be called "internal energy" of the quasi-particle.

\section{Elastic Head-on Collision}

As an illustration, we computed the solution for $\alpha_{2}=0$ (known as Manakov's solution). As expected, no interaction between the two components of the vector soliton was observed, which confirms that only $\alpha_{2}$ governs the nonlinear effects, not the full coefficient $\left(\alpha_{1}+2 \alpha_{2}\right)$. As should have been expected, our computations showed that for $\alpha_{2}=0$, there was no interaction between the two orthogonal modes $\psi$ and $\phi$, despite the fact that $\alpha_{1} \neq 0$ means that terms proportional to $|\psi|^{2}$ are present in the equation for $\phi$, and vice versa.

We chose for the phase speeds of solitons $c_{l}=1$ and $c_{r}=-0.5$ which does not restrict us very much because in absence of linear coupling, $\gamma=\Gamma=0$, one can change the phase speed, but still obtains the same results provided that $\alpha_{1}$ is also changed. The selected values for the phase speeds give for the amplitudes of the initial solitons the following

$$
A_{l} \equiv A_{\psi}=\frac{\sqrt{2}}{2} \approx 0.7075, \quad A_{r} \equiv A_{\phi}=\frac{\sqrt{2}}{4} \approx 0.3537
$$

According to the analytical expression, equation (7), the masses of the two quasiparticles are $M_{l} \equiv M_{\psi}=1$ and $M_{r} \equiv M_{\phi}=0.5$. The total pseudomomentum is $c_{l} M_{l}-c_{r} M_{r}=0.75$. Since at initial time the two QPs are strictly $90^{\circ}$ polarized, we have only one of the amplitudes $A_{\psi}, A_{\phi}$ not equal to zero. Then equation (10) 
can be applied to the left and right solitons separately to get the equalities

$$
\begin{aligned}
& E_{l}^{k}=\frac{1}{2} c_{l}^{2} M_{l}=0.5, \quad E_{l}^{p}=\frac{2}{3}\left(A_{l}^{2} b_{l}-\frac{A_{l}^{4}}{b_{l}}\right)=\frac{2}{3}\left(\frac{1}{4}-\frac{1}{2}\right)=-\frac{1}{6} \approx-0.1667 \\
& E_{l}=E_{l}^{k}+E_{l}^{p}=\frac{1}{3} \approx 0.3333, \quad E_{r}^{k}=\frac{1}{2} c_{r}^{2} M_{r}=\frac{1}{16}=0.0625 \\
& E_{r}^{p}=\frac{2}{3}\left(A_{r}^{2} b_{r}-\frac{A_{r}^{4}}{b_{r}}\right)=\frac{2}{3}\left(\frac{1}{32}-\frac{1}{16}\right)=-\frac{1}{48} \approx-0.0208 \\
& E_{r}=E_{r}^{k}+E_{r}^{p}=\frac{1}{24} \approx 0.0408, \quad E=E_{l}+E_{r}=\frac{3}{8}=0.375
\end{aligned}
$$

where the superscripts $k$ and $p$ stand for "kinetic" and "potential" energies respectively.

Note that the actual values obtained from the initial condition after being discretized on the chosen grid (see the discrete analogues in [7] and [11]), are

$$
M_{l}=1.0000000, M_{r}=0.50000000, P=0.74921909, E=0.37462784 .
$$

The small deviations for $P$ and $E$ of order of $0.1 \%$ are the effect of the truncation error. Since the scheme is conservative, the above values are the one which are kept constant during the time stepping.

\section{Conclusion}

In this paper, we have develop a complex-arithmetic implementation of a conservative difference scheme for vector NLSE.

The new tool developed here allowed us to investigate physically important sets of parameters of the vector NLSE. This means that although the initial conditions are nontrivial for only one of the functions in each of the initial locations, after the interaction both functions can acquire nontrivial amplitudes in both locations.

We consider as an initial profile the superposition of solitons with linear polarizations, one of them having only $\psi$-component, and the other - only $\phi$-component. Then this initial profile is allowed to evolve according to the vector NLSE and the results of the collision depend mostly on the nonlinear coupling parameter $\alpha_{2}$ (cross-modulation parameter) on the dynamics of quasi-particles [11]. The exact formulae for the masses, pseudomomenta, and energy of the initial solitons in the case of initial linear polarization allow to check the validity and relevance of their discrete analogues and to get significant knowledge about the behavior of the interacted soliton envelops considered as quasi-particles. 


\section{Acknowledgements}

MDT is deeply obliged to his dear mentor Professor Christo I. Christov, who had involved him in this subject few years ago and under whose supervision this investigation was started and developed.

The investigation is supported partially by the Scientific Fund of Republic of Bulgaria under Grant DDVU02/71.

\section{References}

[1] Agrawal G., Nonlinear Fiber Optics, $2^{\text {nd }}$ Edn, Academic Press, San Diego 1989.

[2] Agrawal, G., Fiber-Optic Communication Systems, $2^{\text {nd }}$ Edn, Wiley, New York 1997.

[3] Christov C., Dost S., and Maugin G., Inelasticity of Soliton Collisions in System of Coupled NLS Equations, Physica Scripta 50 (1994) 449-454.

[4] Marcuse D., Menyuk C. and Wai P., Application of the Manakov-PMD Equation to Studies of Signal Propagation in Optical Fibers with Randomly Varying Birefringence, J. Lightw. Technol. 15 (1997) 1735-1746.

[5] Manakov S., On the Theory of Two-Dimensional Stationary Self-Focusing of Electromagnetic Waves, Soviet Physics JETP 38 (1974) 248-253.

[6] Menyuk C. and Marks B., Interaction of Polarization Mode Dispersion and Nonlinearity in Optical Fiber Transmission Systems, J. Lightw. Technol. 24 (2006) 2806-2826.

[7] Sonnier W. and Christov C., Strong Coupling of Schrödinger Equations: Conservative Scheme Approach, Mathematics and Computers in Simulation 69 (2005) 514-525.

[8] Sophocleous C. and Parker D., Pulse Colliasions and Polarization Conversion for Optical Fibers, Opt. Commun. 112 (1994) 214-224.

[9] Taha T. and Ablovitz M., Analytical and Numerical Aspects of Certain Nonlinear Evolution Equations II. Numerical, Schrödinger Equation, J. Comp. Phys. 55 (1984) 203-230.

[10] Todorov M. and Christov C., Conservative Scheme in Complex Arithmetic for Coupled Nonlinear Schrödinger Equations, Discrete and Continuous Dynamical Systems, Supl. 2007, pp 982-992.

[11] Todorov M. and Christov C., Impact of the Large Cross-Modulation Parameter on the Collision Dynamics of Quasi-Particles Governed by Vector NLSE, Mathematics and Computers in Simulation 80 (2009) 46-55. 
[12] Zakharov V. and Shabat A., Exact Theory of Two-Dimensional Self-Focusing and One-Dimensional Self-Modulation of Waves in Nonlinear Media, Soviet Physics JETP 34 (1972) 62-9.

[13] Zakharov V. and Shabat A., Interaction Between Solitons in a Stable Medium, Soviet Physics JETP 37 (1973) 823-828.

Michail Todorov

Dept. Applied Math \& Informatics

Technical University of Sofia

1000 Sofia, BULGARIA

E-mail address: mtodetu-sofia.bg 